are exposed to possible presentation bias resulting from the presence of the researcher and from bias inherent in relying upon a small self-selected sample of women who are motivated to participate in research and/or may have a particular interest in GDM. An alternative research approach is to opportunistically use 'found' data that is spontaneously usergenerated within online discussion forums. The objective of this study was to explore the perceptions and beliefs surrounding GDM suggested within online parental-support forums for parents, that could have implications for future T2D prevention.

Methods A search was carried out using the Google search engine to identify online discussion forums that could be of relevance to women with GDM in the UK. We screened these 10 (out of 120 forums) to identify those containing relevant data that could be considered public, and selected Mumsnet and Netmums to be included in the study. They were comprehensively searched using the search term 'gestational diabetes or GD', and relevant posts from 1/1/2017 - 14/2/2019 were identified. Multiple messages from the same person were linked together as a single unit, analogous to a research participant, using an anonymised identifier. A theoretical framework derived previously was used to code and sort the data using framework approach.

Results A total of 646 posts in 137 threads from 282 unique users with current/previous GDM were analysed. Five prominent themes were identified: Emotional response to and understanding of diagnosis; personal responsibility; consequences and impact of GDM; lifestyle change; and Type 2 diabetes. Users' discussions around these themes highlighted three implicit 'messages' regarding GDM: that GDM is not a serious or even real diagnosis and the consequences are not severe; that women need not take personal responsibility for GDM; and there are minimal implications for T2D prevention.

Discussion These (partially) subliminal messages will all mitigate against efforts to encourage women to change their lifestyles for future T2D prevention. This is worrying as online networks have huge reach, and are viewed by millions of visitors who don't necessarily post themselves, but are signposted to these sites when they pose questions to global search engines. The data analysed were generated in a naturalistic setting and are perhaps closer than traditional qualitative research to women's real perceptions and beliefs surrounding GDM.

\section{OP40 EXPLORING BARRIERS AND FACILITATORS FOR WOMEN TO SEEK HELP FOR REPRODUCTIVE SYMPTOMS IN THE UNITED KINGDOM: A RAPID REVIEW}

${ }^{1}$ AA Ayorinde* ${ }^{2} \mathrm{~F}$ Boardman, ${ }^{3} \mathrm{~L}$ Porter, ${ }^{3} \mathrm{~A}$ Hadley, ${ }^{3} \mathrm{M}$ Ludeke, ${ }^{3} \mathrm{~A}$ Sallis, ${ }^{3} \mathrm{~S}$ Mann ${ }^{1}$ ORO Oyebode. 'Warwick Centre for Applied Health Research and Delivery (W-CAHRD), University of Warwick, Coventry, UK; ${ }^{2}$ WMS - Social Science and Systems in Health, University of Warwick, Coventry, UK; ${ }^{3}$ Public Health England, London, UK

\subsection{6/jech-2020-SSMabstracts.40}

Background Many women experience reproductive symptoms that impact on their wellbeing but do not seek help for a range of different reasons. In this rapid review we explored the factors that enable or hinder women to seek help for unwanted or debilitating reproductive symptoms. We also aimed to examine whether existing interventions address these factors and identify opportunities for alternative interventions.

Methods We searched electronic databases MEDLINE, PsycINFO and CINAHL in October 2019 and contacted experts to identify grey literature and additional interventions. The search strategy included terms covering behaviour and intervention combined with terms relating to common reproductive symptoms such as, dysmenorrhoea and menopause. We limited to articles published in English since 2009. We included any study that presented facilitators and barriers to seeking help for reproductive symptoms that was conducted with women in the United Kingdom. We also included articles on relevant interventions. One reviewer screened titles and abstracts with $10 \%$ double screened by a second reviewer. Full texts were screened independently by two reviewers. We extracted study characteristics using a prepiloted form and used the Mixed Methods Appraisal Tool for quality assessment. Data extraction and quality assessment were performed by one reviewer and a sample checked by a second reviewer. We coded information on barriers and facilitators from each study into themes using NVivo. Results were summarised descriptively.

Results We screened 12474 citations, 102 full-text articles and retained 22 articles for analysis. Ten help-articles focused on interventions. Of the 12 articles reporting barriers and facilitators, the most common barriers to seeking help were embarrassment, perception that symptom is too private to talk about, dissatisfaction with the information received from healthcare professionals, poor knowledge, perception that symptom is part of aging and difficulty explaining the symptoms to others. Facilitators were having access to useful information/information pitched at the right level, an information source women felt comfortable with and supportive work environments (including awareness among managers and flexible working).

Conclusion This review identified a range of barriers and facilitators spanning across capability, opportunity and motivation. We have categorised the barriers and facilitators into the Theory and Techniques Tool (TaTT) mechanisms of actions (MoAs). Our next step is to assess whether the existing intervention contents match those deemed theoretically appropriate using TaTT in order to identify missed opportunities for intervention. This will enable us to make recommendations for interventions to support women to seek help for reproductive symptoms when necessary.

\section{OP41 ASSOCIATIONS BETWEEN MODE OF DELIVERY AND OFFSPRING OVERWEIGHT/OBESITY: FINDINGS FROM THE STUDYING LIFECOURSE OBESITY PREDICTORS (SLOPE) POPULATION-BASED COHORT}

${ }^{1}$ A Simpson*, ${ }^{1} \mathrm{G}$ Grove, ${ }^{1} \mathrm{~N}$ Ziauddeen, ${ }^{1,2} \mathrm{NA}$ Alwan. ${ }^{1}$ School of Primary Care, Population Sciences and Medical Education, University of Southampton, Southampton, UK; ${ }^{2}$ NIHR Southampton Biomedical Research Centre, University of Southampton and University Hospital Southampton NHS FT, Southampton, UK

\subsection{6/jech-2020-SSMabstracts.41}

Background Childhood obesity affects one in ten children in England by age 5, and one in five by age 11. Existing evidence suggests a possible relationship between caesarean section (CS) birth and higher risk of overweight/obesity in childhood, however maternal obesity is a strong confounder 
in this relationship. With CS rates rising by $4 \%$ globally per year, we aimed to examine the relationship between mode of delivery and overweight/obesity in childhood.

Methods SLOPE is a linked population-based cohort of anonymised routine antenatal, birth and child healthcare records in Hampshire, UK (2003-2018). Delivery method was categorised into unassisted vaginal delivery, assisted vaginal delivery and CS (including elective and emergency). Child body mass index (BMI) was measured as part of the National Child Measurement Programme in England. Children were identified as overweight/obese if their age- and sex-adjusted BMI was above the 85 th percentile. Generalised linear modelling for outcome at two time points; 4-5 years $(n=30,229)$ and $10-11$ years $(n=14,305)$ was conducted, adjusting for clustering within families. Modelling was introduced in stages with the choice of covariates informed by a Directed Acyclic Graph, first adjusting for maternal BMI, then adding in confounders including maternal age, ethnicity, educational attainment, parity, smoking status at booking appointment, pre-eclampsia, and previous CS (model C) and then birthweight and gestational age at birth as potential mediators (model M). Analyses were also stratified by maternal BMI category (underweight: <18.5, normal weight: 18.5 to $<25$, overweight: 25 to $<30$, obese: $\geq 30$ $\mathrm{kg} / \mathrm{m}^{2}$ ) at booking.

Results Of children delivered by CS, $25.0 \%$ and 33.7\% were overweight/obese by $4-5$ years and $10-11$ years respectively, compared to $21.9 \%$ and $31.0 \%$ respectively with vaginal births. In unadjusted analysis, CS was associated with increased risk of overweight/obesity at 4-5 years (relative risk (RR) 1.13, 95\% Confidence Interval (95\% CI) 1.08-1.19), and at 10-11 years (RR 1.08, 95\% CI 1.02-1.14), however both were attenuated by adjusting for maternal BMI. In stratified analyses, CS delivery was associated with increased risk of childhood overweight/obesity at 4-5 years only in normal weight women (model C: RR 1.15, 95\% CI 1.04-1.27, model M: RR 1.14, 95\% CI 1.02-1.26), but not in 10-11 year models.

Conclusion Maternal weight status at the start of pregnancy is a strong confounder in the relationship between mode of delivery and childhood overweight/obesity. In stratified analyses, this association was evident only for children of normal weight women. If this relationship is causal, the potential mechanisms need to be explored.

\section{OP42 MATERNAL CHARACTERISTICS ASSOCIATED WITH VAPING IN PREGNANCY}

C Opondo*, S Harrison, F Alderdice, C Carson, MA Quigley. Nuffield Department of Population Health, University of Oxford, Oxford, UK

10.1136/jech-2020-SSMabstracts.42

Background The potential for harm to developing foetuses by tobacco products is well established. Studies have focused on cigarette smoking as a source of exposure. The use of electronic cigarettes (vaping devices) has increased in recent years. The Office for National Statistics (ONS) reports vaping data among the general public, with the most recent estimates showing a $5 \%$ prevalence among all women. However, there is no data on prevalence of vaping among pregnant women in the UK. This study sought to estimate the prevalence of vaping during pregnancy and to explore the factors and outcomes associated with vaping in a population-based sample of women giving birth in England.

Methods The study was a cross-sectional population-based postal survey of maternal and infant health and care, the National Maternity Survey (NMS) 2018. A total of 16,000 women aged $16+$ years who were living in England and who had given birth in 2017 were invited to participate. They were identified at random from birth records by the ONS, and surveyed at 6 months post-partum. Demographic and clinical questions about their pregnancy, labour, birth, and postnatal period were sent to them on a questionnaire. We estimated the prevalence of vaping and patterns of cigarette smoking in this group of women, and conducted regression analysis to explore associations between maternal characteristics and vaping, and between vaping and birth outcomes. Survey data were weighted to reduce the potential for nonresponse bias.

Results The crude prevalence of vaping among pregnant women was $2.8 \%$. This varied according to the pattern of cigarette smoking in pregnancy: $0.3 \%$ in never-smokers; $3.3 \%$ in ex-smokers; $7.7 \%$ in women who quit smoking when they became pregnant (pregnancy-inspired quitters); $9.5 \%$ in women who temporarily stopped smoking while pregnant but resumed smoking after the birth of their baby (temporary quitters); and $17.7 \%$ in women who continued to smoke during and after their pregnancy (persistent smokers). Younger women, unmarried women, women with fewer years of formal education, women living with a smoker, and persistent smokers (compared to quitters, ex- and never-smokers) were more likely to vape. Vaping was also associated with 3.2 weeks' reduction in the duration of breastfeeding and $10 \%$ reduction in the prevalence of breastfeeding for at least 8 weeks. However, there was no evidence of association between vaping and any of the maternal characteristics or birth outcomes after adjusting for the pattern of cigarette smoking in pregnancy.

Conclusion The prevalence of vaping among pregnant women in the NMS 2018 survey was low. Characteristics associated with vaping were broadly similar to those associated with smoking. Adverse birth outcomes and reduced breastfeeding among pregnant women who vape may be explained by their relatively higher prevalence of cigarette smoking.

\section{Thursday 10 September}

\section{Hospital Admissions}

\section{OP43 HOW MUCH OF A DOUBLE JEOPARDY ARE PRETERM BIRTH AND SOCIAL DISADVANTAGE? A POPULATION- BASED STUDY OF HOSPITAL ADMISSIONS IN ONE MILLION CHILDREN IN ENGLAND}

${ }^{1} \mathrm{MA}$ Quigley ${ }^{*},{ }^{1} \mathrm{~V}$ Coathup, ${ }^{2} \mathrm{~A}$ Macfarlane, ${ }^{3} \mathrm{~S}$ Johnson, ${ }^{4} \mathrm{~S}$ Petrou, ${ }^{1} \mathrm{O}$ Rivero-Arias, ${ }^{1} \mathrm{C}$ Carson, ${ }^{3} \mathrm{E}$ Boyle, ${ }^{1} \mathrm{JJ}$ Kurinczuk. 'NPEU, Nuffield Department of Population Health, University of Oxford, Oxford, UK; ${ }^{2}$ Department of Health Sciences, City University, London, UK; ${ }^{3}$ Department of Health Sciences, University of Leicester, Leicester, UK; ${ }^{4}$ Nuffield Department of Primary Care Health Sciences, University of Oxford, Oxford, UK

\subsection{6/jech-2020-SSMabstracts.43}

Background Children who are born preterm and those who are socially disadvantaged tend to have increased morbidity in childhood. Few studies are large enough and have 